\title{
Cardiovascular Response During Submaximal Underwater Treadmill Exercise in Stroke Patients
}

\author{
Jeehyun Yoo, MD, Kill-Byung Lim, MD, Hong-Jae Lee, MD, Yong-Geol Kwon, MD
}

Department of Physical Medicine and Rehabilitation, Inje University Ilsan Paik Hospital, Goyang, Korea

\begin{abstract}
Objective To evaluate the cardiovascular response during head-out water immersion, underwater treadmill gait, and land treadmill gait in stroke patients.

Methods Ten stroke patients were recruited for underwater and land treadmill gait sessions. Each session was 40 minutes long; 5 minutes for standing rest on land, 5 minutes for standing rest in water or on treadmill, 20 minutes for treadmill walking in water or on land, 5 minutes for standing rest in water or on treadmill, and 5 minutes for standing rest on land. Blood pressure (BP) and heart rate (HR) were measured during each session. In order to estimate the cardiovascular workload and myocardial oxygen demand, the rate pressure product (RPP) value was calculated by multiplying systolic BP (SBP) by HR.

Results SBP, DBP, mean BP (mBP), and RPP decreased significantly after water immersion, but HR was unchanged. During underwater and land treadmill gait, SBP, mBP, DBP, RPP, and HR increased. However, the mean maximum increases in BP, HR and RPP of underwater treadmill walking were significantly lower than that of land treadmill walking.

Conclusion Stroke patients showed different cardiovascular responses during water immersion and underwater gait as opposed to standing and treadmill-walking on land. Water immersion and aquatic treadmill gait may reduce the workload of the cardiovascular system. This study suggested that underwater treadmill may be a safe and useful option for cardiovascular fitness and early ambulation in stroke rehabilitation.
\end{abstract}

Keywords Blood pressure, Heart rate, Treadmill, Stroke, Rehabilitation

Received April 3, 2014; Accepted June 20, 2014

Corresponding author: Yong-Geol Kwon

Department of Physical Medicine and Rehabilitation, Inje University Ilsan Paik Hospital, 170 Joowha-ro, Ilsanseo-gu, Goyang 411-706, Korea Tel: +82-31-910-7440, Fax: +82-31-910-7746, E-mail: 19480@paik.ac.kr

(c) This is an open-access article distributed under the terms of the Creative Commons Attribution Non-Commercial License (http://creativecommons. org/licenses/by-nc/3.0) which permits unrestricted noncommercial use, distribution, and reproduction in any medium, provided the original work is properly cited.

Copyright $\odot 2014$ by Korean Academy of Rehabilitation Medicine

\section{INTRODUCTION}

Stroke is one of the main causes of long-term disabilities in adults [1]. These physical disabilities include hemiplegia, abnormal gait, abnormal function of the upper limbs, spasticity, joint contracture, pressure ulcer, and decreased cardiopulmonary function. Stroke itself may cause one of the aforementioned physical disabilities; however, the secondary problems from decreased physical activities may cause them to worsen. 
Many treatment methods in the field of rehabilitation have been used to improve the physical condition and cardiovascular function of stroke patients. Among these, treadmill-based gait training is known to be effective in improving the muscle strength of the lower limbs, gait speed, gait endurance, and cardiovascular functions [26]. It is now widely used in clinical practice. The body weight support system may also be used for patients who have abnormal gait pattern after stroke, and cannot maintain their balance during treadmill walking.

Recently, the underwater treadmill has become an alternative for patients who cannot support their weight, or have poor balance after stroke. There are many benefits of using the underwater treadmill due to various water characteristics, such as temperature, viscosity, hydrostatic pressure, turbulence, and drag force [7]. The warm temperature of water can reduce muscular tone of limbs. Furthermore, the viscosity and buoyancy of the water alleviate stress on weight-bearing joints, and can provide postural support for impaired balance. This condition creates a similar environment to treadmill walking with the body weight support system [8]. Water exercise not only has these physiological benefits for the patients but also has various positive psychological effects, such as increased motivation and improved participation. Underwater treadmill walking also enables patients to initiate early gait rehabilitation, providing ambulatory satisfaction in patients who have gait disability and balance impairment.

However, head-out water immersion and underwater exercise may cause various changes in the cardiovascular system $[9,10]$. Exercise generally increases the blood pressure (BP), heart rate (HR), and cardiac output [11]. Head-out water immersion causes the redistribution of the blood volume from the limbs to the thoracic cavity because of hydrostatic pressure and increased cardiac output [12]. This blood translocation can affect the BP and cardiac workload. Moreover, head-out water immersion and underwater exercise are considered to have remarkable effects on the cardiovascular system of stroke patients. The effects of underwater exercise in stroke patients are well known, but the effects on the cardiovascular system are not well established. This study investigated the $\mathrm{BP}, \mathrm{HR}$, and rate pressure product (RPP) during the head-out water immersion and underwater gait, to understand their effects on the cardiovascular system, and compared the results with treadmill walking on land.

\section{MATERIALS AND METHODS}

\section{Subjects}

The subjects for the study were patients who were admitted to our hospital with cerebral infarction or hemorrhage. Inclusion criteria included 1) ability to walk independently or with supporting devices, 2) time required to walk $10 \mathrm{~m}<60$ seconds, 3 ) enough gait endurance to perform the protocol of this study, and 4) patients who do not have fear of falling during walking. Patients with severe heart failure, unstable coronary artery disease, uncontrolled hypertension, or infectious diseases were excluded from the study to avoid the risk of adverse cardiovascular events. Patients with skin inflammation, which was inappropriate for aquatic exercise, were also excluded. A total of 10 patients were analyzed in this study.

\section{Study design and evaluation}

The depth of the water pool was set up to xiphoid process level for each patient. The room temperature was maintained at $25^{\circ} \mathrm{C}-26^{\circ} \mathrm{C}$, while the water temperature was maintained at $34^{\circ} \mathrm{C}-35^{\circ} \mathrm{C}$. The patients were not tested during meal times, and the subjects were asked to take a break on the day after the underwater treadmill walking. The land treadmill walking was performed at the same time on the next day of the underwater treadmill walking. For treadmill gait, AquaGaiter underwater treadmill (Ferno-Washington Inc., Wilmington, $\mathrm{OH}$, USA) and Quasar treadmill (h/p/cosmos Sports \& Medical GmbH, Nussdorf-Traunstein, Germany) were used. Each subject was allowed to walk at his or her own pace.

\section{Underwater treadmill walking}

The systolic BP (SBP), diastolic BP (DBP) and HR of the subjects were measured during standing rest on land (at 1-minute intervals for 5 minutes), standing rest in water (at 1-minute intervals for 5 minutes), treadmill walking in water (at 4-minute intervals for 20 minutes), standing rest in water after walking (at 1-minute intervals for 5 minutes), and standing rest on land (at 1-minute intervals for 5 minutes). Each subject used a gentle slope to enter and leave the water within 30 seconds. The BP cuff was maintained at the level of heart above the water dur- 
ing the experiment.

\section{Land treadmill walking}

The SBP, DBP and HR of the subjects were measured during standing rest on land (at 1-minute intervals for 5 minutes), standing rest on treadmill (at 1-minute intervals for 5 minutes), land treadmill walking (at 4-minute intervals for 20 minutes), standing rest on treadmill after walking (at 1-minute intervals for 5 minutes), and standing rest on land (at 1-minute intervals for 5 minutes).

\section{Measurements}

The SBP, DBP, and HR of the subjects were measured using the automated blood pressure and pulse measuring device (YM1000, Mediana Co. Ltd., Wonju, Korea) on the left upper arm during both sessions. The mean blood pressure $(\mathrm{mBP})$ was calculated as follows: $\mathrm{DBP}+(\mathrm{SBP}-$ DBP)/3. The RPP is correlated with myocardial oxygen consumption. It is obtained by multiplying the SBP and HR in order to evaluate the cardiac workload [13]. The maximum rise of each parameter was determined as the maximum difference of $\mathrm{BP}, \mathrm{HR}$, and RPP between the values before (at the last 1 minute during standing rest on the aquatic treadmill in the water, or on the land treadmill) and during treadmill walking.

\section{Statistical analysis}

The SAS ver. 9.1 for Windows (SAS Institute Inc., Cary, NC, USA) was used for the statistical analysis. The Wilcoxon paired signed-rank test was performed to compare parameters during the treadmill walking, either in the water or on land, to those of the baseline evaluation. The Mann-Whitney U test was used to compare the maximum rise of each parameter during the treadmill walking in the water with the treadmill walking on land. Statistical significance was accepted at $p<0.05$. All values were presented as mean \pm standard deviation.

\section{RESULTS}

Five male and 5 female participants completed the investigation. Their mean age was $48.1 \pm 14.3$ years. Nine of them had cerebral hemorrhage, while one had cerebral infarction (Table 1). Five of them were on hypertension medication, and their blood pressures were well controlled. The SBP, DBP, and RPP were measured while
Table 1. General characteristics of subjects

\begin{tabular}{lc}
\hline Characteristic & Value \\
\hline Gender & \\
\hline Male & 5 \\
\hline Female & 5 \\
Age (yr) & $48.10 \pm 15.26$ \\
\hline Type of stroke & \\
\hline Ischemic & 1 \\
\hline Hemorrhagic & 9 \\
\hline K-MMSE & $21.29 \pm 5.77$ \\
\hline Height $(\mathrm{cm})$ & $161.40 \pm 7.47$ \\
\hline Body weight $(\mathrm{kg})$ & $62.00 \pm 10.41$ \\
\hline BMI $\left(\mathrm{kg} / \mathrm{m}^{2}\right)$ & $23.70 \pm 3.12$ \\
\hline SBP $(\mathrm{mmHg})$ & $121.50 \pm 13.75$ \\
\hline $\mathrm{mBP}(\mathrm{mmHg})$ & $88.30 \pm 8.27$ \\
\hline DBP $(\mathrm{mmHg})$ & $71.70 \pm 7.45$ \\
\hline HR $(\mathrm{beats} / \mathrm{min})$ & $77.10 \pm 6.97$ \\
\hline RPP $(\mathrm{mmHg} / \mathrm{min})$ & $9,367.65 \pm 1,351.97$ \\
\hline Vas & \\
\hline
\end{tabular}

Values are presented as mean \pm standard deviation.

K-MMSE, Korean Mini-Mental State Examination; BMI, body mass index; SBP, systolic blood pressure; $\mathrm{mBP}$, mean blood pressure; DBP, diastolic blood pressure; HR, heart rate; RPP, rate pressure product.

the participants were in a comfortable sitting position in order to evaluate the condition of their cardiovascular systems prior to the study (Table 1). The mean water depth of the pool was $114.50 \pm 6.42 \mathrm{~cm}$. The mean speed of underwater treadmill walking was $1.92 \pm 0.21 \mathrm{~km} / \mathrm{hr}$, while the mean speed of land treadmill walking was $2.26 \pm 0.26$ $\mathrm{km} / \mathrm{hr}$. The change in the cardiovascular system during the exercise was compared with that of the baseline value (at the first 1 minute during standing rest on land), and the results were shown in Figs. 1 and 2. No abnormal cardiovascular responses were observed during or after exercise.

The change in cardiovascular response during underwater treadmill walking

The SBP, DBP, mBP, and RPP decreased significantly for 5 minutes after the head-out water immersion as compared to the baseline, whereas HR did not show a significant change. The SBP, DBP, and $\mathrm{mBP}$ gradually increased while walking. Significantly, the SBP remained below the baseline value for up to 16 minutes of treadmill walking. The HR and RPP also gradually increased during tread- 


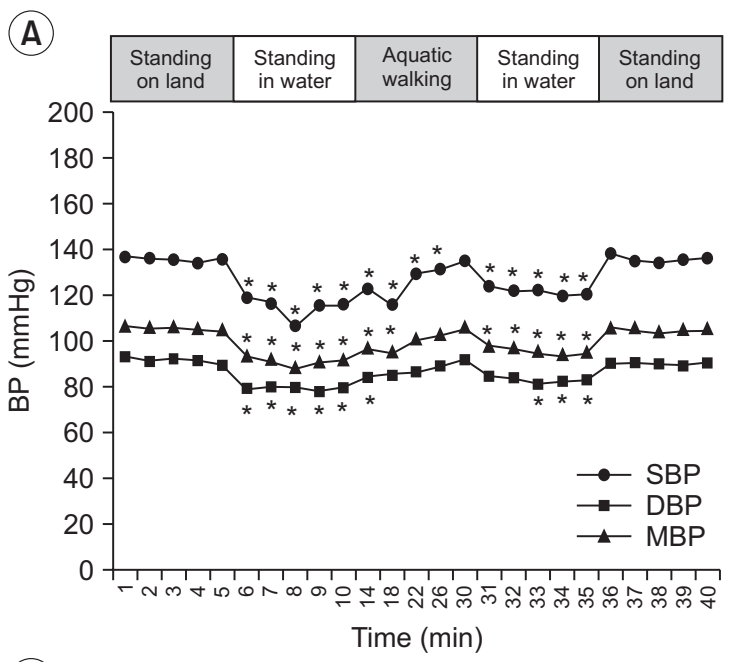

(B)

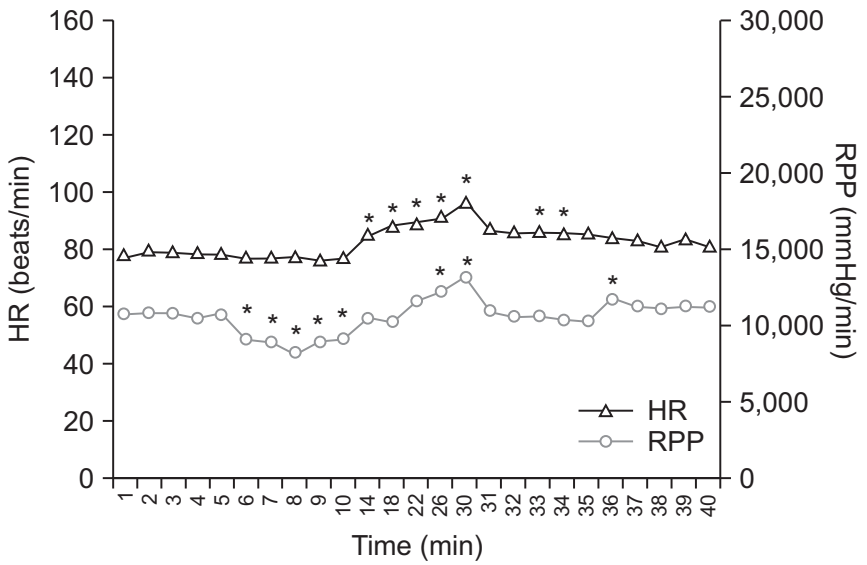

Fig. 1. SBP, $m B P$, and DBP (A), HR and RPP (B) during the aquatic session. $\mathrm{SBP}$, systolic blood pressure; $\mathrm{mBP}$, mean blood pressure; DBP, diastolic blood pressure; HR, heart rate; $\mathrm{RPP}$, rate pressure product. ${ }^{*} \mathrm{p}<0.05$.
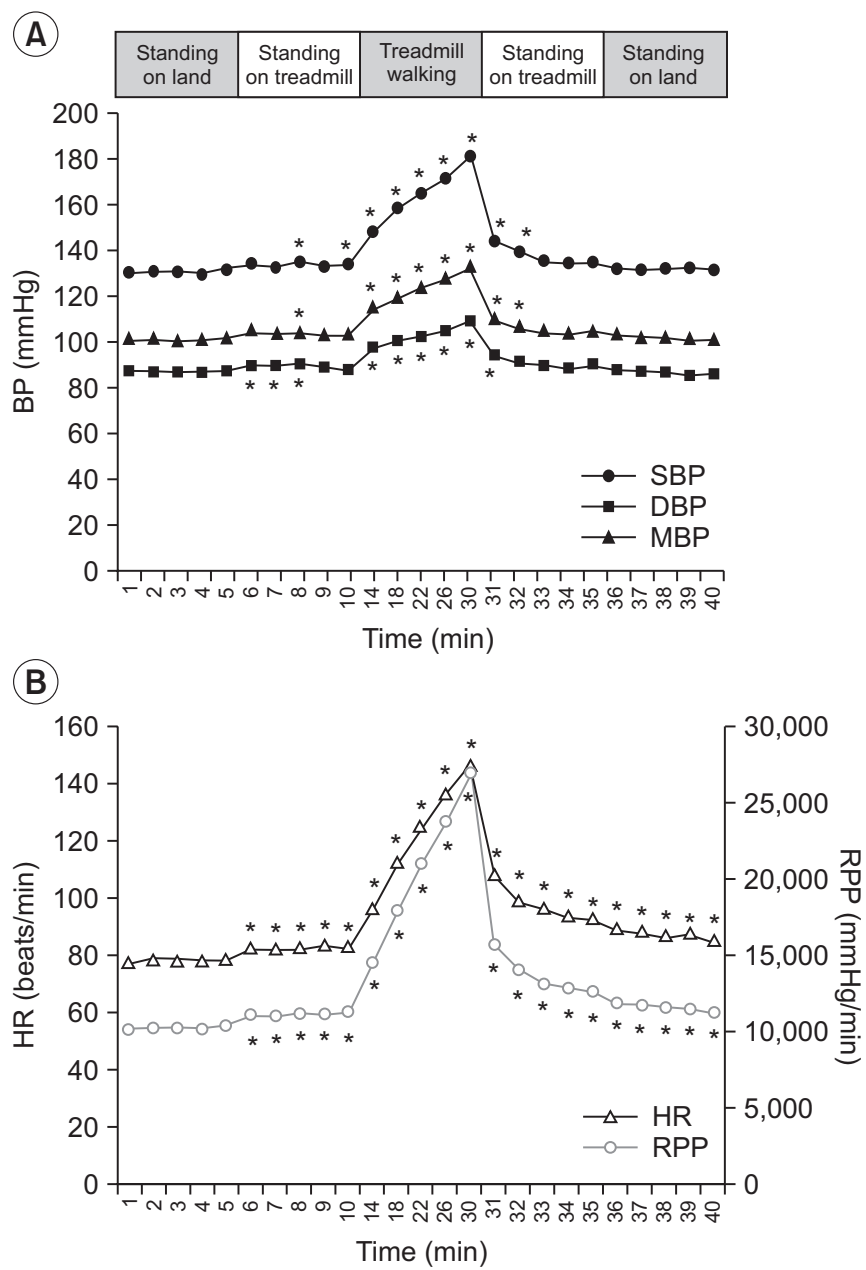

Fig. 2. SBP, $m B P$, and $D B P(A), H R$ and $R P P(B)$ during the land session. $\mathrm{SBP}$, systolic blood pressure; $\mathrm{mBP}$, mean blood pressure; DBP, diastolic blood pressure; HR, heart rate; RPP, rate pressure product. ${ }^{*} \mathrm{p}<0.05$.

Table 2. Means of maximum rises in BP, HR, and DP

\begin{tabular}{lcc} 
& Underwater treadmill & Land treadmill \\
\hline SBP $(\mathrm{mmHg})$ & $18.70 \pm 5.70^{*}$ & $46.70 \pm 19.51$ \\
DBP $(\mathrm{mmHg})$ & $12.30 \pm 8.23^{*}$ & $21.00 \pm 5.40$ \\
$\mathrm{mBP}(\mathrm{mmHg})$ & $14.40 \pm 5.76^{*}$ & $45.00 \pm 29.57$ \\
HR (beats/min) & $18.60 \pm 7.59^{*}$ & $63.50 \pm 27.37$ \\
\hline RPP $(\mathrm{mmHg} / \mathrm{min})$ & $3,981.50 \pm 1,351.36^{*}$ & $15,727.80 \pm 7,357.21$ \\
\hline
\end{tabular}

Values are presented as mean \pm standard deviation.

SBP, systolic blood pressure; mBP, mean blood pressure; DBP, diastolic blood pressure; HR, heart rate; RPP, rate pressure product.

${ }^{*} \mathrm{p}<0.05$.

mill walking, and the change was statistically significant. The mean maximum rises of both SBP and RPP during walking were $18.70 \pm 5.70$ and $3,981.50 \pm 1,351.36$, respec- tively (Table 2). During standing rest in the water after walking, all parameters continuously decreased, and the SBP showed significantly lower level than the baseline 
value. When the participants stood while coming out from the water, the SBP, DBP, and $\mathrm{mBP}$ recovered to the similar level as before water immersion. This change was not statistically significant. The HR and RPP increased as compared to the baseline but the difference was not statistically significant.

The change in cardiovascular response during land treadmill walking

Before the patients started walking on the treadmill, the SBP, DBP, and mBP did not show significant change as compared to the baseline. All parameters rapidly increased during treadmill walking, and the changes were statistically significant. The mean maximum increases of both systolic BP and RPP while walking were $46.70 \pm 19.51$ and $15,727.80 \pm 7,357.21$, respectively (Table 2). After the participants finished walking, the SBP, DBP, mBP gradually decreased, and then recovered to the baseline level. However, the difference from the baseline was not significant. The HR and RPP gradually increased immediately after the participants stood on the treadmill, thereby showing a statistically significant change. This increase was maintained until the end of the land session.

\section{DISCUSSION}

Kelly et al. [14] reported that the cardiorespiratory fitness of subacute stroke patients decreased by $50 \%$ of agematched healthy people, and their walking ability also decreased by $50 \%$ of the healthy group when 6 -minute and 10-minute walking tests were performed. Another study conducted on the cardiopulmonary function of acute stroke patients also showed similar differences, and the mean $\mathrm{VO}_{2}$ peak was only $60 \%$ of that predicted from age- and gender-adjusted normative values for sedentary healthy individuals, thus not meeting physiologic demands of independent living [15]. Various therapeutic methods for improving muscle strength of the paralyzed limb as well as balance and performance of daily activities, should be selected based on the accurate evaluation of their effects on the cardiovascular system with consideration for the decreased physical activities and cardiopulmonary fitness of the stroke patients.

Recently many stroke patients undergo aquatic therapy, and underwater treadmill walking has been implemented in rehabilitation programs for those with difficulty in weight-bearing or poor balance. However, the underwa- ter environment is different from that of air because of the characteristics of water. Because stroke patients have different physical conditions based on their disabilities compared to healthy people, they are expected to show a different level of adaptation to an environmental change. However, there have been no studies on the cardiovascular change of stroke patients in the water. This study evaluated the cardiovascular response in stroke patients during underwater and land treadmill-walking exercises.

During head-out water immersion at thermoneutral temperature $\left(34^{\circ} \mathrm{C}-35^{\circ} \mathrm{C}\right)$, increased surrounding hydrostatic pressure redistributes blood from the dependent limbs to the thoracic cavity. This blood shifting augments cardiac output via increased end-diastolic volume [12,16]. This redistribution of blood volume may affect the cardiac workload and blood pressure. The SBP and DBP increase slightly, and the HR decreases during a short-term immersion [17]. Meanwhile, the BP does not change or decrease slightly when head-out water immersion continues for a few hours [16]. This is due to the decreased activity of the sympathetic nerve system [18] and adaptation mechanism of the cardiovascular system, such as decreased secretion of rennin, angiotensin II, aldosterone, and atrial natriuretic peptide $[19,20]$.

However, the SBP, DBP, mean BP, and RPP of the stroke patients in this study significantly decreased for 5 minutes after head-out water immersion, while their HR did not show any significant change compared to the baseline. This result was opposite to other studies conducted on healthy subjects. A previous study performed on hip or knee arthritis patients also showed similar results as the healthy people. They all had increased SBP, DBP, mean BP, and RPP, but decreased HR [21]. The decreased BP and RPP in this study can be explained by the following 3 reasons. Firstly, room temperature that was maintained at $24^{\circ} \mathrm{C}-25^{\circ} \mathrm{C}$ was different from the water temperature of $34^{\circ} \mathrm{C}-35^{\circ} \mathrm{C}$. The participants stood for 5 minutes outside the pool wearing swimsuits before water immersion. The thermoneutral zone (TNZ) is known as the range of ambient temperatures without regulatory changes in metabolic heat production or evaporative heat loss. The TNZ is affected by the subcutaneous fat, clothing, energy consumption, age, and gender [22]. The room temperature maintained in this study was considered to be lower than the TNZ. Furthermore, as the participants were asked to stand on land for 5 minutes before the water immersion, the sympathetic nerve system might have been 
already activated in order to maintain homeostasis. The increased venous return after the water immersion would stimulate the cardiopulmonary baroreceptors, which can lead to a decrease in the sympathetic tone and systemic vascular resistance $[23,24]$. Therefore, head-out water immersion can decrease activity of the sympathetic nerve system and resistance of the blood vessel. Additional to the difference in the temperature between water and room, previously activated the sympathetic nerve system of the patients could account for an increase in peripheral vascular dilation after water immersion. Secondly, healthy people have a compensation system to increase cardiac muscle contraction and heart rate through the autonomic nerve system and endocrine system for constant cardiac output when the BP decreases for various reasons. However, the participants in this study showed no significant change in $\mathrm{HR}$, while other BP parameters decreased significantly for 5 minutes after water immersion. This implied that stroke patients have decreased function of the autonomic and endocrine systems to maintain homeostasis of the cardiovascular system. This also suggested that they are not well adaptive to the environmental change. Thirdly, the vascular remodeling in the limbs after a stroke may have affected the decrease in the BP. Many studies on stroke patients showed that the lower leg blood flow in the affected limb decreased during rest and exercise [25-27], and the blood flow in the femoral artery of the hemiparetic limb was significantly lower at rest when compared to the other limb [27]. Other studies have also reported that the structural vascular remodeling, characterized by a decreased diameter of vessel and blood flow in the femoral artery of the paralyzed side, may occur after stroke $[26,28]$. The literature suggests that impaired motor function and lower activity level after stroke reduce the needs for blood flow and muscular metabolic activity in the hemiparetic leg [25]. Based on this mechanism, the blood flow to the paralyzed limbs may decrease, thereby affecting the redistribution of the blood volume to the thoracic cavity to some extent, after the head-out water immersion in stroke patients.

Approximately $20 \%$ of stroke survivors remain primarily wheelchair users with gait disability [29]. Gait disability is caused by primary motor impairments that involve paralysis, change in muscle tone, lack of selective motor control, abnormal reflexes, and poor balance. Even though stroke survivors may recover mobility, they still tend to have hemiparetic gait pattern and show altered step length, decreased single limb support time, and change in joint angle of the lower limbs [30-33]. Furthermore, compensatory motor patterns are often observed during the hemiparetic gait, such as the circumduction, vaulting, and pelvic hike. Other problems also include slow walking speed, limited gait endurance, and poor gait stability [34-36]. These changes after stroke lead to inefficient energy expenditure during walking, compared to that of the healthy adult, and may increase the cardiac workload. In this study, the SBP, DBP, mBP, HR, and RPP increased remarkably when the participants performed the treadmill walking on land, and the mean maximum increase of the SBP was $46.70 \pm 19.51 \mathrm{mmHg}$. A previous study showed that physical activities, such as walkinglevel on land, had a positive effect on SBP of about 10 mmHg [37]. Stroke patients in our study comparatively had a greater change in SBP than that of the healthy subjects. This result suggested that cardiovascular workload during walking-level activity in the stroke patients was greater than that of the healthy people.

The SBP, DBP, mBP, and HR gradually increased during the underwater treadmill walking but their mean maximum increases were significantly smaller than those of the land treadmill walking. The RPP result in particular, an indirect indicator of the oxygen consumption of the cardiac muscles, showed a smaller change during the underwater treadmill walking than during the land treadmill walking. The underwater environment decreases the effect of gravity due to the buoyancy and viscosity of water. This helps to decrease the load to the joints and supports the posture of the patients. These characteristics of the underwater environment may relieve many problems caused by hemiparetic gait pattern for stroke patients. This may induce the decrease of energy expenditure and oxygen consumption of the cardiac muscles during walking. The speed of underwater walking was slower than that of the land treadmill walking. However, they experienced more resistance in the water than in ambient air, and walking in chest-deep water yields higher energy costs than walking at similar speeds on land [38]. Thus, the exercise intensity of underwater treadmill walking is similar to that of the land treadmill gait. Also, in consideration for the health condition of the stroke patients, aquatic treadmill walking is an exercise of moderate intensity. Therefore, underwater treadmill walking in stroke patients can better help relieve the cardiovascular workload compared to the land treadmill walking. 
This study did not have an age-matched control group. As a result, the effects of the head-out water immersion and underwater or land treadmill walking on the cardiovascular response could not be compared to the healthy adults directly. Another limitation of this study was the small number of participants. Future studies should be conducted with a larger sample size in order to confirm these results. The study of Asahina et al. [21] on arthritis patients maintained the room and water temperatures to a similar level at $31^{\circ} \mathrm{C}-32^{\circ} \mathrm{C}$. However, this study did not use the same temperature in the water pool and room. The temperature difference may have affected the evaluation values of the study. RPP was used as an indirect indicator of cardiac workload instead of the metabolic gas analysis, because of the limited place and difficulties in using the devices during underwater gait. Lastly, the orders of underwater and land treadmill walking were not randomized. In consideration of the above-mentioned limitations, further studies are required.

In conclusion, this study evaluated the cardiovascular response during the head-out water immersion, and land and underwater treadmill walking in stroke patients. The change in the cardiovascular system after head-out water immersion for stroke patients was different from the results obtained in healthy people, and aquatic treadmill gait may reduce the workload of the cardiovascular system. Therefore, this study suggested that underwater treadmill may be a safe and useful option for cardiovascular fitness and early ambulation in stroke rehabilitation.

\section{CONFLICT OF INTEREST}

No potential conflict of interest relevant to this article was reported.

\section{ACKNOWLEDGMENTS}

This work was supported by the 2011 Inje University research grant.

\section{REFERENCES}

1. Grysiewicz RA, Thomas K, Pandey DK. Epidemiology of ischemic and hemorrhagic stroke: incidence, prevalence, mortality, and risk factors. Neurol Clin 2008;
26:871-95.

2. Ada L, Dean CM, Hall JM, Bampton J, Crompton S. A treadmill and overground walking program improves walking in persons residing in the community after stroke: a placebo-controlled, randomized trial. Arch Phys Med Rehabil 2003;84:1486-91.

3. Macko RF, Ivey FM, Forrester LW, Hanley D, Sorkin JD, Katzel LI, et al. Treadmill exercise rehabilitation improves ambulatory function and cardiovascular fitness in patients with chronic stroke: a randomized, controlled trial. Stroke 2005;36:2206-11.

4. Macko RF, Smith GV, Dobrovolny CL, Sorkin JD, Goldberg AP, Silver KH. Treadmill training improves fitness reserve in chronic stroke patients. Arch Phys Med Rehabil 2001;82:879-84.

5. Patterson SL, Rodgers MM, Macko RF, Forrester LW. Effect of treadmill exercise training on spatial and temporal gait parameters in subjects with chronic stroke: a preliminary report. J Rehabil Res Dev 2008; 45:221-8.

6. Silver KH, Macko RF, Forrester LW, Goldberg AP, Smith GV. Effects of aerobic treadmill training on gait velocity, cadence, and gait symmetry in chronic hemiparetic stroke: a preliminary report. Neurorehabil Neural Repair 2000;14:65-71.

7. Bates A, Hanson N. Aquatic exercise therapy. Philadelphia: Saunders; 1996.

8. Simmons V, Hansen PD. Effectiveness of water exercise on postural mobility in the well elderly: an experimental study on balance enhancement. J Gerontol A Biol Sci Med Sci 1996;51:M233-8.

9. Park KS, Choi JK, Park YS. Cardiovascular regulation during water immersion. Appl Human Sci 1999;18: 233-42.

10. Thompson PD, Franklin BA, Balady GJ, Blair SN, Corrado D, Estes NA 3rd, et al. Exercise and acute cardiovascular events placing the risks into perspective: a scientific statement from the American Heart Association Council on Nutrition, Physical Activity, and Metabolism and the Council on Clinical Cardiology. Circulation 2007;115:2358-68.

11. Iellamo F. Neural mechanisms of cardiovascular regulation during exercise. Auton Neurosci 2001;90:66-75.

12. Arborelius M Jr, Ballidin UI, Lilja B, Lundgren CE. Hemodynamic changes in man during immersion with the head above water. Aerosp Med 1972;43:592-8. 
13. Gobel FL, Norstrom LA, Nelson RR, Jorgensen CR, Wang Y. The rate pressure product as an index of myocardial oxygen consumption during exercise in patients with angina pectoris. Circulation 1978;57:54956.

14. Kelly JO, Kilbreath SL, Davis GM, Zeman B, Raymond J. Cardiorespiratory fitness and walking ability in subacute stroke patients. Arch Phys Med Rehabil 2003;84: 1780-5.

15. MacKay-Lyons MJ, Howlett J. Exercise capacity and cardiovascular adaptations to aerobic training early after stroke. Top Stroke Rehabil 2005;12:31-44.

16. Epstein M. Renal effects of head-out water immersion in humans: a 15-year update. Physiol Rev 1992;72:563621.

17. Gleim GW, Nicholas JA. Metabolic costs and heart rate responses to treadmill walking in water at different depths and temperatures. Am J Sports Med 1989;17:248-52.

18. Razminia M, Trivedi A, Molnar J, Elbzour M, Guerrero $M$, Salem Y, et al. Validation of a new formula for mean arterial pressure calculation: The new formula is superior to the standard formula. Catheter Cardiovasc Interv 2004;63:419-25.

19. Gabrielsen A, Bie P, Holstein-Rathlou NH, Christensen NJ, Warberg J, Dige-Petersen H, et al. Neuroendocrine and renal effects of intravascular volume expansion in compensated heart failure. Am J Physiol Regul Integr Comp Physiol 2001;281:R459-67.

20. Gauer $\mathrm{OH}$. Recent advances in the physiology of whole body immersion. Acta Astronaut 1975;2:31-9.

21. Asahina M, Asahina MK, Yamanaka Y, Mitsui K, Kitahara A, Murata A. Cardiovascular response during aquatic exercise in patients with osteoarthritis. Am J Phys Med Rehabil 2010;98:731-5.

22. Kingma B, Frijns A, van Marken Lichtenbelt W. The thermoneutral zone: implications for metabolic studies. Front Biosci (Elite Ed) 2012;4:1975-85.

23. Mourot L, bouhaddi M, Gandelin E, Cappelle S, Dumoulin G, Wolf JP, et al. Cardiovasuculr autonomic control during short-term thermoneutral and cool head-out immersion. Aviat space Environ Med 2008; 79:14-20.

24. Boussuges A. Immersion in thermoneutral water: effects on arterial compliance. Aviat Space Environ Med 2006;77:1183-7.
25. Ivey FM, Gardner AW, Dobrovolny CL, Macko RF. Unilateral impairment of leg blood flow in chronic stroke patients. Cerebrovasc Dis 2004;18:283-9.

26. Billinger SA, Kluding PM. Use of Doppler ultrasound to assess femoral artery adaptations in the hemiparetic limb in people with stroke. Cerebrovasc Dis 2009; 27:552-8.

27. Landin S, Hagenfeldt L, Saltin B, Wahren J. Muscle metabolism during exercise in hemiparetic patients. Clin Sci Mol Med 1977;53:257-69.

28. Billinger SA, Gajewski BJ, Guo LX, Kluding PM. Single limb exercise induces femoral artery remodeling and improves blood flow in the hemiparetic leg poststroke. Stroke 2009;40:3086-90.

29. Wade DT, Wood VA, Heller A, Maggs J, Langton Hewer R. Walking after stroke: measurement and recovery over the first 3 months. Scand J Rehabil Med 1987;19: 25-30.

30. Wall JC, Turnbull GI. Gait asymmetries in residual hemiplegia. Arch Phys Med Rehabil 1986;67:550-3.

31. Olney SJ, Monga TN, Costigan PA. Mechanical energy of walking of stroke patients. Arch Phys Med Rehabil 1986;67:92-8.

32. Roth EJ, Merbitz C, Mroczek K, Dugan SA, Suh WW. Hemiplegic gait: relationships between walking speed and other temporal parameters. Am J Phys Med Rehabil 1997;76:128-33.

33. Kim CM, Eng JJ. Symmetry in vertical ground reaction force is accompanied by symmetry in temporal but not distance variables of gait in persons with stroke. Gait Posture 2003;18:23-8.

34. von Schroeder HP, Coutts RD, Lyden PD, Billings E Jr, Nickel VL. Gait parameters following stroke: a practical assessment. J Rehabil Res Dev 1995;32:25-31.

35. Newman AB, Haggerty CL, Kritchevsky SB, Nevitt MC, Simonsick EM; Health ABC Collaborative Research Group. Walking performance and cardiovascular response: associations with age and morbidity: the Health, Aging and Body Composition Study. J Gerontol A Biol Sci Med Sci 2003;58:715-20.

36. Patterson SL, Forrester LW, Rodgers MM, Ryan AS, Ivey FM, Sorkin JD, et al. Determinants of walking function after stroke: differences by deficit severity. Arch Phys Med Rehabil 2007;88:115-9.

37. Hayashi T, Ohshige K, Tochikubo O. Exclusion of influence of physical activity on ambulatory blood pres- 
Jeehyun Yoo, et al.

sure. Clin Exp Hypertens 2007;29:23-30.

38. Hall J, Macdonald IA, Maddison PJ, O'Hare JP. Cardiorespiratory responses to underwater treadmill walking in healthy females. Eur J Appl Physiol Occup Physiol 1998;77:278-84. 